\title{
Towards the ultimate SM fit to close in on Higgs physics
}

\author{
Alex Pomarol ${ }^{a}$ and Francesco Riva ${ }^{b}$ \\ ${ }^{a}$ Departament de Física, Universitat Autònoma de Barcelona, \\ 08193 Bellaterra, Barcelona, Spain \\ ${ }^{b}$ Institut de Théorie des Phénomènes Physiques, EPFL, \\ 1015 Lausanne, Switzerland \\ E-mail: pomarol@ifae.es, francesco.riva@epfl.ch
}

ABstRACT: With the discovery of the Higgs at the LHC, experiments have finally addressed all aspects of the Standard Model (SM). At this stage, it is important to understand which windows for beyond the SM (BSM) physics are still open, and which are instead tightly closed. We address this question by parametrizing BSM effects with dimension-six operators and performing a global fit to the SM. We separate operators into different groups constrained at different levels, and provide independent bounds on their Wilson coefficients taking into account only the relevant experiments. Our analysis allows to assert in a modelindependent way where BSM effects can appear in Higgs physics. In particular, we show that deviations from the SM in the differential distributions of $h \rightarrow V \bar{f} f$ are related to other observables, such as triple gauge-boson couplings, and are then already constrained by present data. On the contrary, $B R(h \rightarrow Z \gamma)$ can still hide large deviations from the SM.

Keywords: Higgs Physics, LEP HERA and SLC Physics, Beyond Standard Model

ARXIV EPRINT: 1308.2803 


\section{Contents}

1 Motivation 1

2 Constraining BSM physics from EW observables 2

2.1 Experimental input values 3

$2.2 \quad Z$-pole observables for leptons and the $W$ mass 4

2.3 Quarks 5

$\begin{array}{ll}2.4 & \text { Triple gauge-boson couplings }\end{array}$

3 Higgs physics $\quad 8$

$\begin{array}{ll}3.1 \quad \text { New physics effects in } h \rightarrow V \bar{f} f & 10\end{array}$

4 CP-odd operators $\quad 12$

5 Conclusions 14

A Corrections to $V \bar{f} f$ couplings and $Z$-pole observables $\quad 16$

$\begin{array}{ll}\text { B } q \bar{q}^{\prime} l \bar{\nu} \text { contact-interactions at the LHC } & 17\end{array}$

\section{Motivation}

With the first LHC revenues we have begun to gain experimental evidence for all sectors of the Standard Model (SM). Not having found signs of beyond the SM (BSM) physics, it is time to ask which directions in the parameter space of "deformations" of the SM are still unprobed. This can provide a useful guidance for future experiments.

The purpose of this paper is to take steps towards addressing this question. We will mainly focus on flavor-independent observables, and how these constrain electroweak symmetry breaking (EWSB) effects in gauge-bosons and Higgs physics. As a modelindependent parametrization of BSM physics, we will use the Wilson coefficients of the independent dimension-six operators. This is a valid parametrization as long as the BSM scale is heavier than the electroweak scale, as we will assume here. Instead of an exhaustive fit to the SM, including all available data, our goal is to show which are the most relevant experiments that constrain the different directions in the parameter space of Wilson coefficients, and provide the corresponding bounds.

We will classify the bounds in different groups [1]. First, those arising from $Z$-pole observables, $W$ mass and some low-energy experiments that constrain, at the per-mille level, deviations in $W / Z$ propagators and gauge-boson couplings to fermions. In a second group, we have bounds, a factor $\sim 10$ weaker, from measurements of the triple gauge-boson couplings (TGC), that will be crucial to constrain certain directions in the parameter space, 
left unbound by the first group of experiments. Finally, we will have bounds from Higgs physics arising from the recent LHC data.

One of the main purposes of our fit is to extract indirect constraints on possible deviations from the SM in future Higgs measurements. In particular, we will find that only $\Gamma(h \rightarrow Z \gamma)$ can still hide large deviations from the SM prediction, while deviations in $h \rightarrow V \bar{f} f$ are already constrained by other experiments, as they can be related to TGC measurements. Since the LHC can in principle do better measurements of TGC from processes such as $p p \rightarrow W \gamma, Z \gamma, W W, Z Z$, this will be a more optimal way to constrain BSM physics than from $h \rightarrow V \bar{f} f$ or $V V \rightarrow h$.

There have been several groups also addressing these questions [2-8]. Contrary to ours, however, most analyses have considered operators one by one, setting the rest artificially to zero, and the combined effect of more than one operator is rarely taken into account. The analysis that goes closer in spirit to the one presented here is the one of ref. [2]. Our analysis differs from theirs in several aspects. First of all, our analysis is extended to include Higgs physics and tries to understand where BSM effects can be found in future Higgs measurements. Secondly, and importantly, our choice of basis $[1,9,10]$ allows to separate operators that are constrained at different level. This choice minimizes the correlations between Wilson-coefficient constraints and makes the results easy to interpret. The analysis of ref. [2] was made using the basis of ref. [11], and led to ref. [7] to erroneusly conclude that constraints from electroweak observables were much weaker than what they actually are.

In section 2 we study the constraints on the CP-even Wilson coefficients arising from electroweak (non-Higgs) observables. In section 3 we present the relevant Wilson coefficients for Higgs physics, show their constraints, and study new physics in $h \rightarrow V \bar{f} f$. In section 4 we briefly comment on CP-odd operators. We leave for appendix A the details concerning $Z$-pole observables, and in appendix B we show how LHC data can constrain the relevant four-fermi interactions entering in our analysis.

\section{Constraining BSM physics from EW observables}

We will assume that the BSM sector is heavy, with a mass scale $\Lambda$ much larger than the weak-scale. This allows us to parametrize in a model-independent way all BSM effects by dimension-six operators added to the SM $[11,12]$. There are many options for the choice of the dimension- 6 operator basis. Here we will follow the basis of $[1,10]$ that we find more suitable for our analysis than that of ref. [11], due to its better connection with experiments. Assuming lepton and baryon number conservation, the full set of operators in this basis can be found in tables 1 and 2 of ref. [1]: these contain 5 redundancies that we use to eliminate the 5 operators $\mathcal{O}_{2 W, 2 B, 2 G}$ and $\mathcal{O}_{L}^{l}, \mathcal{O}_{L}^{(3) l}$.

In table 1 we show the dimension- 6 operators of our basis that enter in the observables that we are interested in. We assume minimal flavor violation (MFV) [13], that is to say that the BSM sector respects a $\mathrm{U}(3)^{5}$ flavor-symmetry up to corrections proportional to the SM Yukawa couplings. This allows us to concentrate on flavor-independent constraints. In particular, MFV implies that dipole operator for fermions, as well as $\left(i \tilde{H}^{\dagger} \stackrel{\leftrightarrow}{D_{\mu}} H\right)\left(\bar{u}_{R} \gamma^{\mu} d_{R}\right)$, are proportional to small Yukawa couplings and can then be neglected. In the operators 


\begin{tabular}{|l|}
\hline $\mathcal{O}_{H}=\frac{1}{2}\left(\partial^{\mu}|H|^{2}\right)^{2}$ \\
$\mathcal{O}_{T}=\frac{1}{2}\left(H^{\dagger} \stackrel{\leftrightarrow}{D} H\right)^{2}$ \\
$\mathcal{O}_{6}=\lambda|H|^{6}$ \\
$\mathcal{O}_{W}=\frac{i g}{2}\left(H^{\dagger} \sigma^{a} \stackrel{\leftrightarrow}{D^{\mu}} H\right) D^{\nu} W_{\mu \nu}^{a}$ \\
$\mathcal{O}_{B}=\frac{i g^{\prime}}{2}\left(H^{\dagger} \stackrel{\leftrightarrow}{D^{\mu}} H\right) \partial^{\nu} B_{\mu \nu}$ \\
\hline
\end{tabular}

$$
\begin{aligned}
& \mathcal{O}_{B B}=g^{\prime 2}|H|^{2} B_{\mu \nu} B^{\mu \nu} \\
& \mathcal{O}_{G G}=g_{s}^{2}|H|^{2} G_{\mu \nu}^{A} G^{A \mu \nu} \\
& \mathcal{O}_{H W}=i g\left(D^{\mu} H\right)^{\dagger} \sigma^{a}\left(D^{\nu} H\right) W_{\mu \nu}^{a} \\
& \mathcal{O}_{H B}=i g^{\prime}\left(D^{\mu} H\right)^{\dagger}\left(D^{\nu} H\right) B_{\mu \nu} \\
& \mathcal{O}_{3 W}=\frac{1}{3 !} g \epsilon_{a b c} W_{\mu}^{a \nu} W_{\nu \rho}^{b} W^{c \rho \mu}
\end{aligned}
$$

\begin{tabular}{|l|l|l|}
\hline $\mathcal{O}_{y_{u}}=y_{u}|H|^{2} \bar{Q}_{L} \widetilde{H} u_{R}+$ h.c. & $\mathcal{O}_{y_{d}}=y_{d}|H|^{2} \bar{Q}_{L} H d_{R}+$ h.c. & $\mathcal{O}_{y_{e}}=y_{e}|H|^{2} \bar{L}_{L} H e_{R}+$ h.c. \\
\hline $\mathcal{O}_{R}^{u}=\left(i H^{\dagger} \stackrel{\leftrightarrow}{D_{\mu}} H\right)\left(\bar{u}_{R} \gamma^{\mu} u_{R}\right)$ & $\mathcal{O}_{R}^{d}=\left(i H^{\dagger} \stackrel{\leftrightarrow}{D_{\mu}} H\right)\left(\bar{d}_{R} \gamma^{\mu} d_{R}\right)$ & $\mathcal{O}_{R}^{e}=\left(i H^{\dagger} \stackrel{\leftrightarrow}{D_{\mu}} H\right)\left(\bar{e}_{R} \gamma^{\mu} e_{R}\right)$ \\
$\mathcal{O}_{L}^{q}=\left(i H^{\dagger} \stackrel{\leftrightarrow}{D_{\mu}} H\right)\left(\bar{Q}_{L} \gamma^{\mu} Q_{L}\right)$ & & \\
$\mathcal{O}_{L}^{(3) q}=\left(i H^{\dagger} \sigma^{a} \overleftrightarrow{D_{\mu}} H\right)\left(\bar{Q}_{L} \sigma^{a} \gamma^{\mu} Q_{L}\right)$ & & \\
\hline $\mathcal{O}_{L L}^{(3) q l}=\left(\bar{Q}_{L} \sigma^{a} \gamma_{\mu} Q_{L}\right)\left(\bar{L}_{L} \sigma^{a} \gamma^{\mu} L_{L}\right)$ & & $\mathcal{O}_{L L}^{(3) l}=\left(\bar{L}_{L} \sigma^{a} \gamma^{\mu} L_{L}\right)\left(\bar{L}_{L} \sigma^{a} \gamma_{\mu} L_{L}\right)$ \\
\hline
\end{tabular}

Table 1. Set of CP-even dimension-6 operators that defines our basis. We are including only the four-fermion operators that affect our observables. We omit dipole operators for fermions and $\mathcal{O}_{R}^{u d}=y_{u}^{\dagger} y_{d}\left(i \tilde{H}^{\dagger} \stackrel{\leftrightarrow}{D_{\mu}} H\right)\left(\bar{u}_{R} \gamma^{\mu} d_{R}\right)$ since they are suppressed by light fermion Yukawas under the MFV assumption. Also $\mathcal{O}_{3 G}$ is not included since it does not enter in our observables. The complete set of operators can be found in ref. [1].

of table 1 we assume a contraction of family indices $i, j$ inside each parenthesis, e.g., $\left(\bar{Q}_{L} \gamma^{\mu} Q_{L}\right)=\left(\bar{Q}_{L}^{i} \gamma^{\mu} Q_{L}^{i}\right)$, and $y_{u} \bar{Q}_{L} u_{R}=y_{u}^{i j} \bar{Q}_{L}^{i} u_{R}^{j}$, as implied by the MFV assumption at the leading order in a Yukawa expansion. The top quark, having a large Yukawa coupling, could depart from the MFV assumption. For this reason we will also consider the impact of treating top operators separately.

CP-odd operators are not included in table 1, since they do not interfere with the SM contributions to the observables that we are considering. Their Wilson coefficients only enter quadratically in these processes and can then be neglected at the linear level that we are working. In section 4 , however, we will briefly discuss their implications for TGC and $h \rightarrow V \bar{f} f$.

\subsection{Experimental input values}

We take the SM as defined by the 3 parameters $g, g^{\prime}$ and $v \simeq 246 \mathrm{GeV}$, that we relate with the well-measured values of the Fermi constant $G_{F}$ as measured in muon decays, the fine-structure constant $\alpha_{\mathrm{em}}$, and the $Z$-boson mass $m_{Z}$. New physics, parametrized through the dimension- 6 operators of table 1 , affects these 3 input observables. The subset of relevant operators is

$$
\Delta \mathcal{L}_{\text {input }}=\frac{c_{T}}{v^{2}} \mathcal{O}_{T}+\frac{c_{V}^{+}}{m_{W}^{2}}\left(\mathcal{O}_{W}+\mathcal{O}_{B}\right)+\frac{c_{L L}^{(3) l}}{v^{2}} \mathcal{O}_{L L}^{(3) l}
$$

where we have defined

$$
c_{V}^{ \pm}=\frac{1}{2}\left(c_{W} \pm c_{B}\right)
$$


and $c_{W, B}$ are the Wilson coefficients of the operators $\mathcal{O}_{W, B}$. The orthogonal combination $\mathcal{O}_{W}-\mathcal{O}_{B}$ does not affect the input parameters. Notice that, instead of a common suppression scale $\Lambda$, we are suppressing the operators of eq. (2.1) by the weak scale, either $m_{W}$ or $v$, meaning that we have absorbed the dependence on $\Lambda$ into the Wilson coefficients. With this normalization the Wilson coefficients must be considered smaller than $O(1)$ for our expansion to make sense.

The modifications of the 3 input observables due to the dimension- 6 operators are given by

$$
\frac{\delta \alpha_{\mathrm{em}}}{\alpha_{\mathrm{em}}}=-2 s_{\theta_{W}}^{2} \hat{S}, \quad \frac{\delta m_{Z}^{2}}{m_{Z}^{2}}=-\hat{T}+2 s_{\theta_{W}}^{2} \hat{S}, \quad \frac{\delta G_{F}}{G_{F}}=-2 c_{L L}^{(3) l},
$$

where we have defined $s_{\theta_{W}} \equiv \sin \theta_{W}$ (and similarly for other trigonometric functions), being $\theta_{W}$ the weak mixing angle, and

$$
\hat{S}=2 c_{V}^{+}, \quad \hat{T}=c_{T},
$$

characterize the dominant contributions to the $W / Z$ propagators $[14,15]$.

Our fits for electroweak observables are performed using a $\chi^{2}$ analysis; the experimental data including correlations, are taken from refs. [16, 17], while the SM predictions, including the dominant loop corrections, are taken from refs. [18, 19] (we have checked that our results including only $\hat{S}$ and $\hat{T}$ agree with ref. [18] at the percent level). Henceforth when referring to SM predictions to observables we will always be considering predictions given as a function of these 3 input experimental values, $G_{F}, \alpha_{\mathrm{em}}$ and $m_{Z}$.

\section{$2.2 \quad Z$-pole observables for leptons and the $W$ mass}

Among the most accurate experimental tests of the SM, stand the $Z$-pole observables measured at LEP-I/SLC, and the Tevatron measurement of the $W$ mass. In a first set of constraints, we single out the leptonic observables that are measured at the per-mille level. The relevant dimension- 6 operators that contribute to these quantities are, apart from $\Delta \mathcal{L}_{\text {input }}$,

$$
\Delta \mathcal{L}_{\text {leptons }}=\frac{c_{R}^{e}}{v^{2}} \mathcal{O}_{R}^{e}
$$

It is important to notice that, being at the $Z$-pole, four-fermion operators can be neglected. LEP-I and SLC measurements afford only 3 observables for the lepton sector, that we can think of as $\Gamma_{Z}^{l_{L}} \equiv \Gamma\left(Z \rightarrow \bar{l}_{L} l_{L}\right), \Gamma_{Z}^{l_{R}} \equiv \Gamma\left(Z \rightarrow \bar{l}_{R} l_{R}\right)$ and $\Gamma_{Z}^{\nu} \equiv \Gamma(Z \rightarrow \bar{\nu} \nu)$. These can be extracted from the correlated set of observables $A_{l}, R_{l}, \sigma_{\text {had }}^{0}, \Gamma_{Z}$ defined in eq. (A.6) of appendix A, where $\Gamma_{Z}^{\nu}=\Gamma_{Z}-\Gamma_{Z}^{\text {visible }}$ since we assume that there are no extra light degrees of freedom. The modifications of these quantities with respect to the SM predictions due to the dimension- 6 operators are given by (see appendix A for details)

$$
\begin{aligned}
\frac{\delta \Gamma_{Z}^{\nu}}{\Gamma_{Z}^{\nu}} & =\hat{T}-\frac{\delta G_{F}}{G_{F}}, \quad \frac{\delta \Gamma_{Z}^{l_{L}}}{\Gamma_{Z}^{l_{L}}}=\frac{1}{c_{2 \theta_{W}}^{2}}\left(\hat{T}-\frac{\delta G_{F}}{G_{F}}-4 s_{\theta_{W}}^{2} \hat{S}\right), \\
\frac{\delta \Gamma_{Z}^{l_{R}}}{\Gamma_{Z}^{l_{R}}} & =-\frac{1}{c_{2 \theta_{W}}^{2}}\left(\hat{T}-\frac{\delta G_{F}}{G_{F}}-2 \hat{S}\right)-\frac{c_{R}^{e}}{s_{\theta_{W}}^{2}} .
\end{aligned}
$$


This gives 3 observables to constrain 4 Wilson coefficients. As an additional observable needed to constrain all 4 coefficients, we take the $W$ mass, whose correction from the SM value is given by

$$
\frac{\delta m_{W}}{m_{W}}=\frac{1}{2 c_{2 \theta_{W}}}\left[c_{\theta_{W}}^{2} \hat{T}-s_{\theta_{W}}^{2}\left(\frac{\delta G_{F}}{G_{F}}+2 \hat{S}\right)\right] .
$$

Using this subset of observables, we can fit $c_{T}, c_{V}^{+}, c_{R}^{e}$ and $c_{L L}^{(3) l}$. Marginalizing over all but one coefficient at a time we find the $95 \%$ C.L. intervals:

$$
\begin{array}{lr}
c_{T} \in[-5,1] \times 10^{-3}, & c_{V}^{+} \in[-6,0] \times 10^{-3}, \\
c_{R}^{e} \in[-5,0] \times 10^{-3}, & c_{L L}^{(3) l} \in[-12,2] \times 10^{-3} .
\end{array}
$$

Other four-lepton operators, apart from $\mathcal{O}_{L L}^{(3) l}$, can be constrained by LEP-II measurements of the cross-sections $e^{+} e^{-} \rightarrow l^{+} l^{-}$[20]. These operators and their experimental constraints are completely orthogonal to our analysis and can be studied independently. We will not pursue them further.

\subsection{Quarks}

Experimental data for quark physics abounds. The most accurate measurements come, again, from LEP-I physics at the $Z$-pole. Assuming flavor-universality, the relevant dimension-6 operators for this type of physics are, apart from $\Delta \mathcal{L}_{\text {input }}$,

$$
\Delta \mathcal{L}_{\text {quarks }}=\frac{c_{L}^{q}}{v^{2}} \mathcal{O}_{L}^{q}+\frac{c_{L}^{(3) q}}{v^{2}} \mathcal{O}_{L}^{(3) q}+\frac{c_{R}^{u}}{v^{2}} \mathcal{O}_{R}^{u}+\frac{c_{R}^{d}}{v^{2}} \mathcal{O}_{R}^{d}
$$

Eq. (2.10) contains 4 new parameters that need 4 new observables in order to be constrained. We take $R_{b}, \Gamma_{Z}^{\text {had }}, A_{b}$ and $A_{c}$ as defined in appendix A. They are affected by dimension- 6 operators as

$$
\begin{aligned}
\frac{\delta \Gamma_{Z}^{\mathrm{had}}}{\Gamma_{Z}^{\mathrm{had}}} & \simeq 0.7 c_{L}^{q}+2.3 c_{L}^{(3) q}+0.3 c_{R}^{u}-0.2 c_{R}^{d}-1.5 \delta s_{\theta_{W}}^{2}+\hat{T}-\frac{\delta G_{F}}{G_{F}} \\
\frac{\delta R_{b}}{R_{b}} & \simeq 1.6 c_{L}^{q}-0.05 c_{L}^{(3) q}-0.3 c_{R}^{u}-0.1 c_{R}^{d}+0.2 \delta s_{\theta_{W}}^{2} \\
\frac{\delta A_{c}}{A_{c}} & \simeq-0.9 c_{L}^{q}+0.9 c_{L}^{(3) q}-2.3 c_{R}^{u}-4.2 \delta s_{\theta_{W}}^{2} \\
\frac{\delta A_{b}}{A_{b}} & \simeq 0.1 c_{L}^{q}+0.1 c_{L}^{(3) q}+0.8 c_{R}^{d}-0.6 \delta s_{\theta_{W}}^{2}
\end{aligned}
$$

where $\delta s_{\theta_{W}}^{2}$ is the correction to the effective weak mixing angle:

$$
\delta s_{\theta_{W}}^{2}=\frac{1}{c_{2 \theta_{W}}}\left[s_{\theta_{W}}^{2} \hat{S}-\frac{s_{2 \theta_{W}}^{2}}{4}\left(\hat{T}-\frac{\delta G_{F}}{G_{F}}\right)\right] .
$$

Only the first two observables, $\Gamma_{Z}^{\text {had }}$ and $R_{b}$, are measured at the per-mille level [16] and can give strong constraints on $c_{L}^{(3) q}$ and $c_{L}^{q}$, since, accidentally, they enter respectively with large coefficients in eq. (2.11) and eq. (2.12). 
The above hadronic observables can be complemented with information from lowenergy measurements. One of the most relevant, due to its high accuracy, is the determination of the unitarity of the CKM matrix by KLOE and $\beta$-decays [21]. This result can be interpreted as a measurement of the Fermi constant in quark-lepton weak interactions, $G_{F}^{(q)}$, normalized to $G_{F}$ as extracted from $\mu$-decays:

$$
\left(\frac{G_{F}^{(q)}}{G_{F}}\right)^{2}=0.9999(12) \quad \text { at } 95 \% \text { C.L. . }
$$

Dimension-six operators modify this SM prediction:

$$
\delta\left(\frac{G_{F}^{(q)}}{G_{F}}\right)^{2}=4 c_{L L}^{(3) l}+2 c_{L}^{(3) q}-2 c_{L L}^{(3) q l},
$$

which also includes a contribution from 4-fermion operators:

$$
\Delta \mathcal{L}_{\text {quark-lepton }}=\frac{c_{L L}^{(3) q l}}{v^{2}} \mathcal{O}_{L L}^{(3) q l}
$$

Now, using recent LHC data [22] for the measurement of the high-energy differential crosssections of $\bar{q} q \rightarrow \bar{l} \nu$, we can independently constrain $c_{L L}^{(3)} q l$, as shown in appendix B. This allows us to use eq. (2.17) to put an independent constraint on $c_{L}^{(3) q}$. Putting all the above information together and marginalizing (since the observables eqs. (2.11)-(2.14) are also sensitive to $\Delta \mathcal{L}_{\text {input }}$, we must include also the leptonic operators and observables and perform a global fit: the resulting constraints on the coefficients of $\Delta \mathcal{L}_{\text {input }}$ will be given in the conclusion), we obtain the $95 \%$ C.L. intervals:

$$
\begin{array}{rlrl}
c_{L}^{q} & \in[-1,4] \times 10^{-3}, & c_{L}^{(3) q} & \in[-7,4] \times 10^{-3}, \\
c_{R}^{u} & \in[-8,0] \times 10^{-3}, & c_{R}^{d} & \in[-53,1] \times 10^{-3}, \\
c_{L L}^{(3)} q l & \in[-2,3] \times 10^{-3} . &
\end{array}
$$

It is interesting to consider the case in which operators made of the top quark have different coefficients from those associated to operators made with the first two families. This is motivated by the largeness of the top Yukawa coupling that suggests that there can be large deviations from flavor-universality. We then add the following operator to our analysis: ${ }^{1}$

$$
\Delta \mathcal{L}_{\text {top }}=\frac{c_{L}^{+q_{3}}}{v^{2}}\left(\mathcal{O}_{L}^{q_{3}}+\mathcal{O}_{L}^{(3) q_{3}}\right), \quad \text { where } c_{L}^{+q_{3}}=\frac{1}{2}\left(c_{L}^{q_{3}}+c_{L}^{(3) q_{3}}\right),
$$

that, due to the presence of $b_{L}$ inside the 3 rd family doublet $Q_{L}$, gives an extra contribution to the observables of eqs. $(2.11)-(2.14)$ :

$$
\left.\frac{\delta \Gamma_{Z}^{\mathrm{had}}}{\Gamma_{Z}^{\mathrm{had}}}\right|_{q_{3}} \simeq 0.5 c_{L}^{+q_{3}},\left.\quad \frac{\delta R_{b}}{R_{b}}\right|_{q_{3}} \simeq 1.8 c_{L}^{+q_{3}},\left.\quad \frac{\delta A_{b}}{A_{b}}\right|_{q_{3}} \simeq 0.1 c_{L}^{+q_{3}} .
$$

\footnotetext{
${ }^{1}$ The other operators involving top-quarks, $\left(\mathcal{O}_{L}^{q_{3}}-\mathcal{O}_{L}^{(3) q_{3}}\right)$ and $\mathcal{O}_{R}^{t}$, affect only top couplings to gauge bosons and their constraints arise from top physics at the LHC. We will not discuss them here.
} 
We have now 5 coefficients in the quark sector, eq. (2.10) and eq. (2.20), but also 5 observables, those of eqs. (2.11)-(2.14) and eq. (2.16). KLOE data, which in the flavor-universal case was only used to slightly improve the numerical constraints, is now crucial to avoid unconstrained directions. The analysis gives: ${ }^{2}$

$$
c_{L}^{+q_{3}} \in[-7,13] \times 10^{-3} .
$$

Other four-quark operators, apart from eq. (2.17), can be constrained by LHC di-jet measurements $[23,24]$. As for the lepton case, these bounds do not interfere with our analysis, neither are relevant for Higgs physics; for this reason they can be studied in a separate context.

\subsection{Triple gauge-boson couplings}

Extra important experimental data needed to further constrain our set of operators is the measure of the $Z W W$ and $\gamma W W$ couplings. The best measurements still come from $e^{+} e^{-} \rightarrow W^{+} W^{-}$at LEP-II, although LHC data is starting to be competitive. The dimension-6 operators involved in this process are, apart from eqs. (2.1) and (2.5),

$$
\Delta \mathcal{L}_{\mathrm{TGC}}=\frac{\kappa_{H V}^{+}}{m_{W}^{2}}\left(\mathcal{O}_{H W}+\mathcal{O}_{H B}\right)+\frac{c_{V}^{-}+\kappa_{H V}^{-}}{2 m_{W}^{2}} \mathcal{O}_{+}+\frac{\kappa_{3 W}}{m_{W}^{2}} \mathcal{O}_{3 W},
$$

where we have defined

$$
\kappa_{H V}^{ \pm}=\frac{1}{2}\left(\kappa_{H W} \pm \kappa_{H B}\right),
$$

with $\kappa_{H W, H B}$ the Wilson coefficients of $\mathcal{O}_{H W, H B}$, and

$$
\mathcal{O}_{ \pm} \equiv\left(\mathcal{O}_{W}-\mathcal{O}_{B}\right) \pm\left(\mathcal{O}_{H W}-\mathcal{O}_{H B}\right)
$$

Notice that in order to connect with the different experiments, we are, for convenience, working with the orthogonal combinations $\left\{\mathcal{O}_{W}+\mathcal{O}_{B}, \mathcal{O}_{H W}+\mathcal{O}_{H B}, \mathcal{O}_{ \pm}\right\}$instead of the original subset $\left\{\mathcal{O}_{W}, \mathcal{O}_{B}, \mathcal{O}_{H W}, \mathcal{O}_{H B}\right\}$ given in table 1 . It is easy to show that the coefficients of $\mathcal{O}_{ \pm}$are given by the combinations $\left(c_{V}^{-} \pm \kappa_{H V}^{-}\right) / 2$. In eq. (2.23) we have not included the operator $\mathcal{O}_{-}$since it does not enter in TGC, and it is only relevant for Higgs physics as we will see later. Indeed, one finds the following contributions to the $Z W W$ and $\gamma W W$ vertices [25]:

$$
\begin{aligned}
\Delta \mathcal{L}_{3 V}= & i g \delta g_{1}^{Z} c_{\theta_{W}} Z^{\mu}\left(W^{-\nu} W_{\mu \nu}^{+}-W^{+\nu} W_{\mu \nu}^{-}\right)+i g\left(\delta \kappa_{Z} c_{\theta_{W}} Z^{\mu \nu}+\delta \kappa_{\gamma} s_{\theta_{W}} A^{\mu \nu}\right) W_{\mu}^{-} W_{\nu}^{+} \\
& +\frac{i g}{m_{W}^{2}}\left(\lambda_{Z} c_{\theta_{W}} Z^{\mu \nu}+\lambda_{\gamma} s_{\theta_{W}} A^{\mu \nu}\right) W_{\nu}^{-\rho} W_{\rho \mu}^{+}
\end{aligned}
$$

where $V_{\mu \nu} \equiv \partial_{\mu} V_{\nu}-\partial_{\nu} V_{\mu}$ for $V=W^{ \pm}, Z, A$, and

$$
\begin{aligned}
\delta g_{1}^{Z} & =\frac{c_{W}+\kappa_{H W}}{c_{\theta_{W}}^{2}}=\frac{1}{c_{\theta_{W}}^{2}}\left(c_{V}^{+}+c_{V}^{-}+\kappa_{H V}^{-}+\kappa_{H V}^{+}\right), \\
\delta \kappa_{\gamma} & =\kappa_{H W}+\kappa_{H B}=2 \kappa_{H V}^{+}, \quad \delta \kappa_{Z}=\delta g_{1}^{Z}-t_{\theta_{W}}^{2} \delta \kappa_{\gamma}, \\
\lambda_{Z} & =\lambda_{\gamma}=\kappa_{3 W} .
\end{aligned}
$$

\footnotetext{
${ }^{2}$ Introducing this extra parameter slightly alters the limits of eqs. (2.9) and (2.19), as we will show later.
} 
Notice that out of the 5 quantities in eq. (2.26), only 3 are independent if one considers only dimension-6 operators; we take these to be $\delta g_{1}^{Z}, \delta \kappa_{\gamma}$ and $\lambda_{\gamma}$. This means that data from the differential cross-sections of $e^{+} e^{-} \rightarrow W^{+} W^{-}$can only constrain 3 extra combinations of Wilson coefficients that corresponds to those of eq. (2.23): $\kappa_{H V}^{+}, c_{V}^{-}+\kappa_{H V}^{-}$ and $\kappa_{3 W} \cdot{ }^{3}$ Unfortunately a three-parameter analysis has not been provided by the full LEP-II collaboration [17, 20], but only by DELPHI [26]. Nonetheless, a small value $\kappa_{3 W}$ is expected in most theories where the SM gauge bosons are assumed to be elementary above the BSM scale $\Lambda$. Neglecting this contribution (and then taking $\lambda_{\gamma}=0$ ), we can use the two-parameter fit of $g_{1}^{Z}$ and $\kappa_{\gamma}$ from ref. [17], to obtain the $95 \%$ C.L. intervals:

$$
c_{V}^{-}+\kappa_{H V}^{-} \in[-4.4,6.6] \times 10^{-2}, \quad \kappa_{H V}^{+} \in[-5.5,3.9] \times 10^{-2} .
$$

Including in the fit $\kappa_{3 W}$ does not change considerably our results; using DELPHI data [26] (which is however strongly correlated with ref. [17]) one finds results similar to eq. (2.28) and $\kappa_{3 W} \in[-4,7] \times 10^{-2}$.

\section{Higgs physics}

We now extend our analysis to Higgs physics. Apart from the operators already introduced before that could also affect Higgs physics, we have $8 \mathrm{CP}$-even dimension-six operators that give contributions only to Higgs physics and not to other SM processes. These are those operators that can be built from the Higgs modulus, i.e., $|H|^{2}$. In our basis, table 1, these operators are

$$
\Delta \mathcal{L}_{\text {Higgs }}=\frac{c_{H}}{v^{2}} \mathcal{O}_{H}+\sum_{f=t, b, \tau} \frac{c_{y_{f}}}{v^{2}} \mathcal{O}_{y_{f}}+\frac{c_{6}}{v^{2}} \mathcal{O}_{6}+\frac{\kappa_{B B}}{m_{W}^{2}} \mathcal{O}_{B B}+\frac{\kappa_{G G}}{m_{W}^{2}} \mathcal{O}_{G G}+\frac{c_{V}^{-}-\kappa_{H}^{-}}{2 m_{W}^{2}} \mathcal{O}_{-}
$$

which holds for one family, taken to be the 3rd one as it is the most relevant for Higgs physics. The presence of $\mathcal{O}_{-}$must be traced back [1] to the fact that the operator $\mathcal{O}_{W W}=$ $g^{2}|H|^{2} W^{\mu \nu a} W_{\mu \nu}^{a}$, that can obviously affect only Higgs physics, when written in our basis is given by

$$
\mathcal{O}_{W W}=4 \mathcal{O}_{-}+\mathcal{O}_{B B}
$$

Now, the operator $\mathcal{O}_{6}$ is in one-to-one correspondence with a deviation in the triple-Higgs coupling that has not yet been measured at the LHC. Therefore, we will not be considering it any longer. The 7 remaining operators of eq. (3.1) affect the main Higgs branching ratios, as well as Higgs production cross-sections at the LHC. In particular, we have that the 4 Wilson coefficients $c_{y_{b}}, c_{y_{\tau}}, \kappa_{B B}, \kappa_{H V}^{-}$enter respectively in $B R(h \rightarrow b b), B R(h \rightarrow \tau \tau)$, $B R(h \rightarrow \gamma \gamma), B R(h \rightarrow Z \gamma)$, while $\kappa_{G G}$ affects $\sigma(G G \rightarrow h)$; the coefficient $c_{H}$ gives a contribution to the Higgs propagator and then enters universally in all Higgs processes (affecting, in particular, vector-boson fusion), while $c_{y t}$ modifies the $h \bar{t} t$ coupling that enters indirectly in $B R(h \rightarrow \gamma \gamma)$ and $B R(h \rightarrow Z \gamma)$ at the one-loop level [9], and also enters in

\footnotetext{
${ }^{3}$ Other Wilson coefficients entering in the process $e^{+} e^{-} \rightarrow W^{+} W^{-}$, such as those of eqs. (2.1) and (2.5), can be neglected since their constraints, derived in our previous analysis, are stronger than the ones we will be obtaining here.
} 
the associated production process $p p \rightarrow h \bar{t} t$ that the LHC can become sensitive to in the near future. The Lagrangian for these processes is determined by

$$
\mathcal{L}_{h}^{\mathrm{SM}}+\Delta \mathcal{L}_{h}
$$

where

$$
\mathcal{L}_{h}^{\mathrm{SM}}=\frac{1}{2}\left(\partial_{\mu} h\right)^{2}+\left(\sqrt{2} G_{F}\right)^{1 / 2} h\left[-m_{f}\left(\bar{f}_{L} f_{R}+\text { h.c. }\right)+m_{Z}^{2} Z_{\mu} Z^{\mu}+2 m_{W}^{2} W_{\mu}^{+} W^{-\mu}\right]+\cdots,
$$

gives the SM contribution to single-Higgs processes written as a function of the physical masses, $m_{f}, m_{Z}$ and $m_{W}^{2}=m_{Z}^{2}\left(1+\sqrt{1-2^{3 / 2} \pi \alpha_{\mathrm{em}} / m_{Z}^{2} G_{F}}\right) / 2$, while

$$
\begin{aligned}
\Delta \mathcal{L}_{h}= & \frac{c_{H}}{2}\left(\partial^{\mu} h\right)^{2}+\frac{h}{v}\left[\delta g_{h f f}\left(\bar{f}_{L} f_{R}+\text { h.c. }\right)+\delta g_{h Z Z} Z_{\mu} Z^{\mu}+\delta g_{h W W} W_{\mu}^{+} W^{-\mu}\right. \\
& \left.+4 \kappa_{B B} s_{\theta_{W}}^{2} A_{\mu \nu} A^{\mu \nu}+4 \kappa_{G G} \frac{g_{s}^{2}}{g^{2}} G_{\mu \nu}^{A} G^{\mu \nu} A+4 t_{\theta_{W}} \kappa_{Z \gamma} Z_{\mu \nu} A^{\mu \nu}\right]
\end{aligned}
$$

gives the contributions proportional to the Wilson coefficients of $\Delta \mathcal{L}_{\text {input }}, \Delta \mathcal{L}_{\text {Higgs }}$, and also that of $\mathcal{O}_{+}$in eq. (2.23) that enters in $h Z \gamma$. The first line of eq. (3.5) includes the corrections to the SM $h \bar{f} f$ and $h V V$ interactions $(V=W, Z)$ due to the Wilson coefficients entering in the input parameters, in the wave-function renormalization of $V$ and in direct contributions arising from $\mathcal{O}_{T}$. They read

$$
\frac{\delta g_{h Z Z}}{g_{h Z Z}^{\mathrm{SM}}}=-c_{T}-\frac{\delta G_{F}}{2 G_{F}}, \quad \frac{\delta g_{h W W}}{g_{h W W}^{\mathrm{SM}}}=2 \frac{\delta m_{W}}{m_{W}}-\frac{\delta G_{F}}{2 G_{F}}, \quad \frac{\delta g_{h f f}}{g_{h f f}^{\mathrm{SM}}}=-c_{y_{f}}-\frac{\delta G_{F}}{2 G_{F}} .
$$

With the exception of $c_{y_{f}}$, we know from the analysis of section 2 that these effects are small. The second line of eq. (3.5) gives the corrections to $h \gamma \gamma, h G G$ and $h Z \gamma$ coupling, where

$$
\kappa_{Z \gamma}=-\frac{1}{2} \kappa_{H V}^{-}-2 s_{\theta_{W}}^{2} \kappa_{B B} .
$$

The dominant modifications from eq. (3.5) to $B R(h \rightarrow \bar{f} f), B R(h \rightarrow \gamma \gamma), B R(h \rightarrow$ $Z \gamma)$ and $\sigma(G G \rightarrow h)$ can be found in [9]. We confront these with a combination of ATLAS [27] and CMS [28-30] data (for technical details see ref. [31]). For this fit we also include the measurements of $p p \rightarrow h \rightarrow V V^{*}$ and vector-boson fusion $p p \rightarrow V V q q \rightarrow h q q$; there are extra contributions to these processes, as we will see in the next subsection (eqs. (3.9) and (3.10)), but these, we can advance, are found to be small and can be neglected here. Strong bounds can be found for Wilson coefficients entering at tree-level in the effective Higgs couplings to $G G, \gamma \gamma$ and $Z \gamma$, as they arise in the SM at the one-loop level. Indeed, marginalizing over $c_{H}$ and $c_{y_{f}}$, we obtain at the $95 \%$ C.L.

$$
\kappa_{G G} \in[-0.8,0.8] \times 10^{-3}, \quad \kappa_{B B} \in[-1.3,1.8] \times 10^{-3}, \quad \kappa_{Z \gamma} \in[-6,12] \times 10^{-3} .
$$

Notice that the constraints on $g_{S}^{2} \kappa_{G G}$ are comparable to those on $g^{\prime 2} \kappa_{B B}$, and that the bound on the $h Z_{\gamma}$ coupling is quite strong even though the experimental data is only sensitive to $B R(h \rightarrow Z \gamma)$ that are $\sim 10$ above the $\mathrm{SM}$ value [32,33]. For the Wilson 
coefficients $c_{H}$ and $c_{y_{f}}$ we do not find significant bounds after marginalizing over the others (for this reason we do not report them). Although this implies that there is room for BSM here, we recall that these coefficients cannot be larger than one if we want our expansion (in higher-dimensional operators) to be reliable.

This ends our analysis for getting the most important constraints on the operators of table 1. Our results can be particularly useful to bound possible new physics effects in future new Higgs measurements. We show this below with the example of the $h \rightarrow V \bar{f} f$ decay.

\subsection{New physics effects in $h \rightarrow V \bar{f} f$}

The decays $h \rightarrow V \bar{f} f(V=W, Z)$ are potentially much richer than two-body decays, since the different differential partial-widths can give in principle extra information on BSM contributions [7, 34-39]. Nevertheless, as we will show, most of the new information that we could extract from measuring the various differential partial-widths of the decay $h \rightarrow V \bar{f} f$ is already constrained by other experiments.

Contributions to $h \rightarrow V \bar{f} f$ can come from corrections to $h V V$ vertices and contactinteractions $h V \bar{f} f$. Apart from the contributions given in eq. (3.3), we have

$$
\begin{aligned}
\Delta \mathcal{L}_{h V V}= & 2 \frac{h}{v}\left[\hat{c}_{W}\left(W_{\mu}^{-} \mathcal{D}^{\mu \nu} W_{\nu}^{+}+\text {h.c. }\right)+\hat{c}_{Z} Z_{\mu} \mathcal{D}^{\mu \nu} Z_{\nu}+\left(\hat{c}_{W}-\hat{c}_{B}\right) t_{\theta_{W}} Z_{\mu} \mathcal{D}^{\mu \nu} A_{\nu}\right] \\
& -2 \frac{h}{v}\left[c_{W W} W^{+\mu \nu} W_{\mu \nu}^{-}+c_{Z Z} Z^{\mu \nu} Z_{\mu \nu}\right] \\
\Delta \mathcal{L}_{h V f f}= & \frac{h}{v} \sum_{f=f_{L}, f_{R}}\left[g_{h W f f^{\prime}} W_{\mu} \bar{f} \gamma^{\mu} f^{\prime}+g_{h Z f f} Z_{\mu} \bar{f} \gamma^{\mu} f\right]
\end{aligned}
$$

where $\mathcal{D}_{\mu \nu}=\partial_{\mu} \partial_{\nu}-\square \eta_{\mu \nu}$ and

$$
\begin{aligned}
\hat{c}_{W} & =c_{W}+\kappa_{H W}, & \hat{c}_{Z} & =\hat{c}_{W}+\hat{c}_{B} t_{\theta_{W}}^{2}, \\
c_{W W} & =\kappa_{H W}, & c_{Z Z} & =\frac{1}{2}\left(\kappa_{H W}+\kappa_{H B} t_{\theta_{W}}^{2}\right)-2 \frac{s_{\theta_{W}}^{4}}{c_{\theta_{W}}^{2}} \kappa_{B B} .
\end{aligned}
$$

Eq. (3.10) gives the contributions to the contact $h V \bar{f} f$ vertices that is found to be correlated with those to the $V \bar{f} f$ vertices:

$$
g_{h Z f f}=\frac{2}{v} \delta g_{Z}^{f} \quad \text { and } \quad g_{h W f f^{\prime}}=\frac{2}{v} \delta g_{W}^{f},
$$

where $\delta g_{Z}^{f}$ and $\delta g_{W}^{f}$ are given respectively in eqs. (A.3) and (A.11) of appendix A.

The CP-even part of the total amplitude for the process $h \rightarrow V \bar{f} f$ can be written as ${ }^{4}$

$$
\mathcal{M}\left(h \rightarrow V J_{f}\right)=\left(\sqrt{2} G_{F}\right)^{1 / 2} \epsilon^{* \mu}(q) J_{f}^{V \nu}(p)\left[A_{f}^{V} \eta_{\mu \nu}+B_{f}^{V}\left(p \cdot q \eta_{\mu \nu}-p_{\mu} q_{\nu}\right)\right],
$$

where $q$ and $p$ are respectively the total 4-momentum of $V$ and the fermion pair in the $J_{f}^{V}$ current $\left(J_{f_{L, R}}^{\mu}=\bar{f}_{L, R} \gamma^{\mu} f_{L, R}\right), \epsilon^{\mu}$ is the polarization 4-vector of $V$, and we have defined

$$
A_{f}^{V}=a_{f}^{V}+\widehat{a}_{f}^{V} \frac{p^{2}+m_{V}^{2}}{p^{2}-m_{V}^{2}}, \quad B_{f}^{V}=b_{f}^{V} \frac{1}{p^{2}-m_{V}^{2}}+\widehat{b}_{f}^{V} \frac{1}{p^{2}} \quad\left(\widehat{b}_{f}^{V}=0 \text { for } V=W\right) .
$$

\footnotetext{
${ }^{4}$ We neglect terms proportional to the light fermion masses (see however ref. [38]). Also we omit a term proportional to $C_{f}^{W} \epsilon_{\mu \nu \alpha \beta} p^{\alpha} q^{\beta}$ that could be CP-even if $C_{f}^{W}$ is pure imaginary. None of the Wilson coefficients of the dimension- 6 operators contribute to this term at tree-level.
} 
The above coefficients are in one-to-one correspondence with the coefficients of the Lagrangians eqs. (3.5), (3.9) and (3.10):

$$
\begin{array}{ll}
a_{f}^{Z}=-g_{Z}^{f}\left(1+\frac{\delta g_{h Z Z}}{m_{Z}^{2}}\right)+2 e Q_{f}\left(\hat{c}_{W}-\hat{c}_{B}\right) t_{\theta_{W}}+v g_{h Z f f}, & a_{f}^{W}=-g_{W}^{f}\left(1+\frac{\delta g_{h W W}}{2 m_{W}^{2}}\right)+v g_{h W f f^{\prime}}, \\
\widehat{a}_{f}^{Z}=g_{Z}^{f}\left(1+\frac{\delta g_{h Z Z}}{m_{Z}^{2}}+2 \hat{c}_{Z}\right), & \widehat{a}_{f}^{W}=g_{W}^{f}\left(1+\frac{\delta g_{h W W}}{2 m_{W}^{2}}+2 \hat{c}_{W}\right), \\
b_{f}^{Z}=8 g_{Z}^{f} c_{Z Z}, & b_{f}^{W}=4 g_{W}^{f} c_{W W}, \\
\widehat{b}_{f}^{Z}=-8 e Q_{f} t_{\theta_{W}} \kappa_{Z \gamma}, &
\end{array}
$$

where we have not included the universal contribution from $c_{H}$ that drops when calculating $B R$. All contributions to the 7 quantities in eq. (3.16) correspond to Wilson coefficients that have already been constrained by other experiments. Indeed, the terms proportional to $\delta g_{h V V}$ of eq. (3.6) and the universal part of the shifts in $g_{V}^{f}$, given in eqs. (A.4) and (A.11), are constrained, as we have shown, at the per-mil level, and can be readily neglected. Similarly, $\kappa_{B B}$ that appears in eq. (3.12) is constrained from $B R(h \rightarrow \gamma \gamma)$. The contributions proportional to $g_{h Z f f}$ that depends, in particular, on $c_{R}^{u, d}$, are also constrained but, as shown in eq. (2.19), only at the per-cent level. Nevertheless, their effects in total amplitudes squared, when summed over the different flavors, can be constrained much more. This is due to the interesting relation

$$
\sum_{f=\text { quarks }} g_{Z}^{f} g_{h Z f f}=\frac{2}{v} \sum_{f=\text { quarks }} g_{Z}^{f} \delta g_{Z}^{f}=\frac{1}{v} \frac{\delta \Gamma_{Z}^{\text {had }}}{\Gamma_{Z}^{\text {had }}} \sum_{f=\text { quarks }}\left(g_{Z}^{f}\right)^{2}+O\left(\hat{S}, \hat{T}, \delta G_{F} / G_{F}\right)
$$

where in the first equality we have used eq. (3.13) and in the second we have used the hadronic part of eq. (A.9) that is true up to pieces proportional to $\hat{S}, \hat{T}$ and $\delta G_{F} / G_{F}$. Since all quantities on the right-hand side of eq. (3.17) are very well measured (per-mille level), we obtain a constraint on the effect of $g_{h Z f f}$ at this level. Neglecting these terms, we are left with only 3 Wilson coefficients, $c_{V}^{-}$and $\kappa_{H V}^{ \pm}$, that are constrained by the measurements of the $Z(\gamma) W W$ and $h Z \gamma$ couplings and whose bounds are not so strong. Using eq. (2.27) and eq. (3.7), we can relate the 7 coefficients of eq. (3.16) with the 3 experimental values of $\delta g_{1}^{Z}, \delta \kappa_{\gamma}$ and $\kappa_{Z \gamma}$. The result is

$$
\begin{array}{ll}
a_{f}^{Z} \simeq-g_{Z}^{f, \mathrm{SM}}+2 e Q_{f}\left(s_{2 \theta_{W}} \delta g_{1}^{Z}-t_{\theta_{W}} \delta \kappa_{\gamma}\right), & a_{f}^{W} \simeq-g_{W}^{f, \mathrm{SM}}, \\
\widehat{a}_{f}^{Z} \simeq g_{Z}^{f, \mathrm{SM}}\left(1+2 c_{2 \theta_{W}} \delta g_{1}^{Z}+2 t_{\theta_{W}}^{2} \delta \kappa_{\gamma}\right), & \widehat{a}_{f}^{W}=g_{W}^{f, \mathrm{SM}}\left(1+2 c_{\theta_{W}}^{2} \delta g_{1}^{Z}\right), \\
b_{f}^{Z} \simeq \frac{2 g_{Z}^{f, \mathrm{SM}}}{c_{\theta_{W}}^{2}}\left(\delta \kappa_{\gamma}-4 c_{2 \theta_{W}} \kappa_{Z \gamma}\right), & b_{f}^{W} \simeq 2 g_{W}^{f, \mathrm{SM}}\left(\delta \kappa_{\gamma}-4 \kappa_{Z \gamma}\right), \\
\widehat{b}_{f}^{Z} \simeq-8 e Q_{f} t_{\theta_{W}} \kappa_{Z \gamma} . &
\end{array}
$$

We can use the experimental constraints on $\delta g_{1}^{Z}, \delta \kappa_{\gamma}$ and $\kappa_{Z \gamma}$ to put a constraint on the 
size of these 7 quantities. For example, for the case of $h \rightarrow Z \bar{l} l$, we find

$$
\begin{aligned}
& \frac{\delta a_{l_{L}}^{Z}}{a_{l_{L}}^{Z}} \in[-0.2,0.1], \quad \frac{\delta \widehat{a}_{l_{L}}^{Z}}{\widehat{a}_{l_{L}}^{Z}} \in[-8,7] \times 10^{-2}, \quad b_{l_{L}}^{Z} \in[-2,5] \times 10^{-2}, \quad \widehat{b}_{l_{L}}^{Z} \in[-2,5], \times 10^{-2}, \\
& \frac{\delta a_{l_{R}}^{Z}}{a_{l_{R}}^{Z}} \in[-0.2,0.3], \quad \frac{\delta \widehat{a}_{l_{R}}^{Z}}{\widehat{a}_{l_{R}}^{Z}} \in[-8,7] \times 10^{-2}, \quad b_{l_{R}}^{Z} \in[-3,2] \times 10^{-2}, \quad \widehat{b}_{l_{R}}^{Z} \in[-2,5] \times 10^{-2} .
\end{aligned}
$$

Although the allowed range in $a_{l_{L, R}}^{Z}$ is quite large, we notice that their impact on the total amplitude, when summed over lepton chiralities, is much smaller, $2 \sum_{l=l_{L}, l_{R}} g_{Z}^{l} a_{l}^{Z} / \sum_{l=l_{L}, l_{R}}\left(g_{Z}^{l}\right)^{2} \in[-6,4] \times 10^{-2}$.

It is interesting to notice that in the limit $g^{\prime} \rightarrow 0$ the result of eq. (3.18) is custodial invariant, i.e., one finds equal corrections for $Z$ and $W$. This is because, for $g^{\prime} \rightarrow 0$, the only Wilson coefficients breaking the custodial symmetry are $c_{T}$ and $c_{L, R}^{f}[1]$ that, being constrained at the per-mille, have been dropped from eq. (3.18). We then find that the test of the custodial symmetry used at LHC [40] defined as

$$
\lambda_{W Z}^{2} \equiv \frac{\Gamma\left(h \rightarrow W W^{(*)}\right)}{\Gamma^{\mathrm{SM}}\left(h \rightarrow W W^{(*)}\right)} \frac{\Gamma^{\mathrm{SM}}\left(h \rightarrow Z Z^{(*)}\right)}{\Gamma\left(h \rightarrow Z Z^{(*)}\right)},
$$

is constrained by

$$
\begin{aligned}
\lambda_{W Z}^{2}-1 & \simeq s_{\theta_{W}}^{2}\left[0.9 c_{W}-2.6 c_{B}+3 \kappa_{H W}-3.9 \kappa_{H B}\right] \\
& \simeq 0.6 \delta g_{1}^{Z}-0.5 \delta \kappa_{\gamma}-1.6 \kappa_{Z \gamma} \in[-6,8] \times 10^{-2},
\end{aligned}
$$

where the numerical values of the first line have been taken from [5]. We see that eq. (3.20) puts a bound on $\lambda_{W Z}$ much stronger than the present direct experimental limit given by $[27]:^{5}\left(\lambda_{W Z}-1\right) \in[-0.5,0.1]$.

Along the lines presented here we could also study the corrections from Wilson coefficients to the Higgs production modes $\bar{f} f \rightarrow V h$ and $V V \rightarrow h$ that we could similarly show that are constrained by our previous analysis.

\section{CP-odd operators}

For completeness, we show here how CP-odd operators enter in TGC and in the process $h \rightarrow V \bar{f} f$, and how they can be related. These operators are $^{6}$

$$
\begin{array}{rlrl}
\mathcal{O}_{B \widetilde{B}} & =g^{\prime 2}|H|^{2} B_{\mu \nu} \widetilde{B}^{\mu \nu}, & & \mathcal{O}_{G \widetilde{G}}=g_{s}^{2}|H|^{2} G_{\mu \nu}^{A} \widetilde{G}^{A \mu \nu}, \\
\mathcal{O}_{H \widetilde{W}}=i g\left(D^{\mu} H\right)^{\dagger} \sigma^{a}\left(D^{\nu} H\right) \widetilde{W}_{\mu \nu}^{a}, & & \mathcal{O}_{H \widetilde{B}}=i g^{\prime}\left(D^{\mu} H\right)^{\dagger}\left(D^{\nu} H\right) \\
\mathcal{O}_{3 \widetilde{W}}=\frac{1}{3 !} g \epsilon_{a b c} W_{\mu}^{a \nu} W_{\nu \rho}^{b} \widetilde{W}^{c \rho \mu}, & &
\end{array}
$$

\footnotetext{
${ }^{5}$ The experimental bound on $\lambda_{W Z}$ is extracted not only using eq. (3.19) but also considering custodial breaking effects in vector-boson fusion. The impact of these latter effects is however negligible.

${ }^{6} \mathrm{~A}$ CP-odd operator involving 3 gluon field-strengths and the operators $i y_{f}|H|^{2} \bar{f}_{L} H f_{R}+$ h.c. complete the list of CP-odd operators; since they do not enter in the observables discussed here, they have been omitted.
} 
where $\widetilde{V}^{\mu \nu}=\epsilon^{\mu \nu \rho \sigma} V_{\rho \sigma} / 2$ for $V=W, B, G$. These operators affect TGC as [25]:

$\Delta \mathcal{L}_{3 \widetilde{V}}=i g\left(\delta \widetilde{\kappa}_{Z} c_{\theta_{W}} \widetilde{Z}^{\mu \nu}+\delta \widetilde{\kappa}_{\gamma} s_{\theta_{W}} \widetilde{A}^{\mu \nu}\right) W_{\mu}^{-} W_{\nu}^{+}+\frac{i g}{m_{W}^{2}}\left(\widetilde{\lambda}_{Z} c_{\theta_{W}} \widetilde{Z}^{\mu \nu}+\widetilde{\lambda}_{\gamma} s_{\theta_{W}} \widetilde{A}^{\mu \nu}\right) W_{\nu}^{-\rho} W_{\rho \mu}^{+}$,

where

$$
\delta \widetilde{\kappa}_{\gamma}=\kappa_{H \widetilde{W}}+\kappa_{H \widetilde{B}}, \quad \delta \widetilde{\kappa}_{Z}=-t_{\theta_{W}}^{2} \delta \widetilde{\kappa}_{\gamma}, \quad \widetilde{\lambda}_{Z}=\widetilde{\lambda}_{\gamma}=\kappa_{3 \widetilde{W}} .
$$

Although the experimental collaborations have not devoted explicit searches for these types of structures, constraints on the above coefficients can be obtained in a near future. For example, ref. [41] finds that with $10 \mathrm{fb}^{-1}$ the $14 \mathrm{TeV}$ LHC can potentially obtain $\left|\delta \widetilde{\kappa}_{\gamma}\right| \lesssim 0.06$.

The contribution from CP-odd operators to Higgs physics can be read from the Lagrangian

$$
\begin{aligned}
\Delta \mathcal{L}_{h V \widetilde{V}}= & -2 \frac{h}{v}\left[c_{W \widetilde{W}} W^{+\mu \nu} \widetilde{W}_{\mu \nu}^{-}+c_{Z \widetilde{Z}} Z^{\mu \nu} \widetilde{Z}_{\mu \nu}-2 t_{\theta_{W}} \widetilde{\kappa}_{Z \gamma} A^{\mu \nu} \widetilde{Z}_{\mu \nu}\right. \\
& \left.-2 \kappa_{B \widetilde{B}} s_{\theta_{W}}^{2} A_{\mu \nu} \widetilde{A}^{\mu \nu}-2 \kappa_{G \widetilde{G}} \frac{g_{s}^{2}}{g^{2}} G_{\mu \nu}^{A} \widetilde{G}^{\mu \nu A}\right]
\end{aligned}
$$

where, quite analogously to the CP-even case, we have

$c_{W \widetilde{W}}=\kappa_{H \widetilde{W}}, \quad c_{Z \widetilde{Z}}=\frac{1}{2}\left(\kappa_{H \widetilde{W}}+\kappa_{H \widetilde{B}} t_{\theta_{W}}^{2}\right)-2 \frac{s_{\theta_{W}}^{4}}{c_{\theta_{W}}^{2}} \kappa_{B \widetilde{B}}, \widetilde{\kappa}_{Z \gamma}=\frac{1}{4}\left(\kappa_{H \widetilde{B}}-\kappa_{H \widetilde{W}}\right)-2 s_{\theta_{W}}^{2} \kappa_{B \widetilde{B}}$.

Finally, the CP-odd contribution to the $h \rightarrow V \bar{f} f$ amplitude is given by

$$
\mathcal{M}\left(h \rightarrow V J_{f}\right)=\left(\sqrt{2} G_{F}\right)^{1 / 2} \epsilon^{* \mu}(q) J_{f}^{V \nu}(p)\left[C_{f}^{V} \epsilon_{\mu \nu \alpha \beta} p^{\alpha} q^{\beta}\right],
$$

where we find [42]

$$
C_{f}^{V}=c_{f}^{V} \frac{1}{p^{2}-m_{V}^{2}}+\widehat{c}_{f}^{V} \frac{1}{p^{2}} \quad\left(\widehat{c}_{f}^{V}=0 \text { for } V=W\right)
$$

with

$$
\begin{aligned}
& c_{f}^{Z}=8 g_{Z}^{f} c_{Z \widetilde{Z}}, \\
& c_{f}^{W}=4 g_{W}^{f} c_{W \widetilde{W}}, \\
& \widehat{c}_{f}^{Z}=-8 e Q_{f} t_{\theta_{W}} \widetilde{\kappa}_{Z \gamma} .
\end{aligned}
$$

Using eq. (4.2) and eq. (4.4), we find the relations

$$
c_{f}^{Z}=\frac{2 g_{Z}^{f}}{c_{\theta_{W}}^{2}}\left(\delta \widetilde{\kappa}_{\gamma}-4 c_{2 \theta_{W}} \widetilde{\kappa}_{Z \gamma}-2 s_{2 \theta_{W}}^{2} \kappa_{B \widetilde{B}}\right), \quad c_{f}^{W}=2 g_{W}^{f}\left(\delta \widetilde{\kappa}_{\gamma}-4 \widetilde{\kappa}_{Z \gamma}-8 s_{\theta_{W}}^{2} \kappa_{B \widetilde{B}}\right) \text {. }
$$

To our knowledge, the only CP-odd observables measured at present are those in the decay $h \rightarrow Z Z^{*} \rightarrow 4 l$ [29], but only very weak constraints can be extracted for the respective Wilson coefficients. Nevertheless, the experimental bound on $B R(h \rightarrow Z \gamma)$ and $B R(h \rightarrow \gamma \gamma)$ can also be used to constrain the squared of $\widetilde{\kappa}_{Z \gamma}$ and $\kappa_{B \widetilde{B}} \cdot{ }^{7}$ One obtains similar constraints to their CP-even counterparts, since the interference term involves the SM contribution that is one-loop suppressed. Neglecting $\widetilde{\kappa}_{Z \gamma}$ and $\kappa_{B \widetilde{B}}$ in eq. (4.9), one obtains a one-to-one relation between the CP-odd contributions to $h \rightarrow V \bar{f} f$ and the TGC parameter $\delta \widetilde{\kappa}_{\gamma}$ that can be measured in the near future.

\footnotetext{
${ }^{7}$ The CP-odd and CP-even contributions add quadratically in the Higgs branching ratios, giving then independent bounds on the corresponding Wilson coefficients.
} 

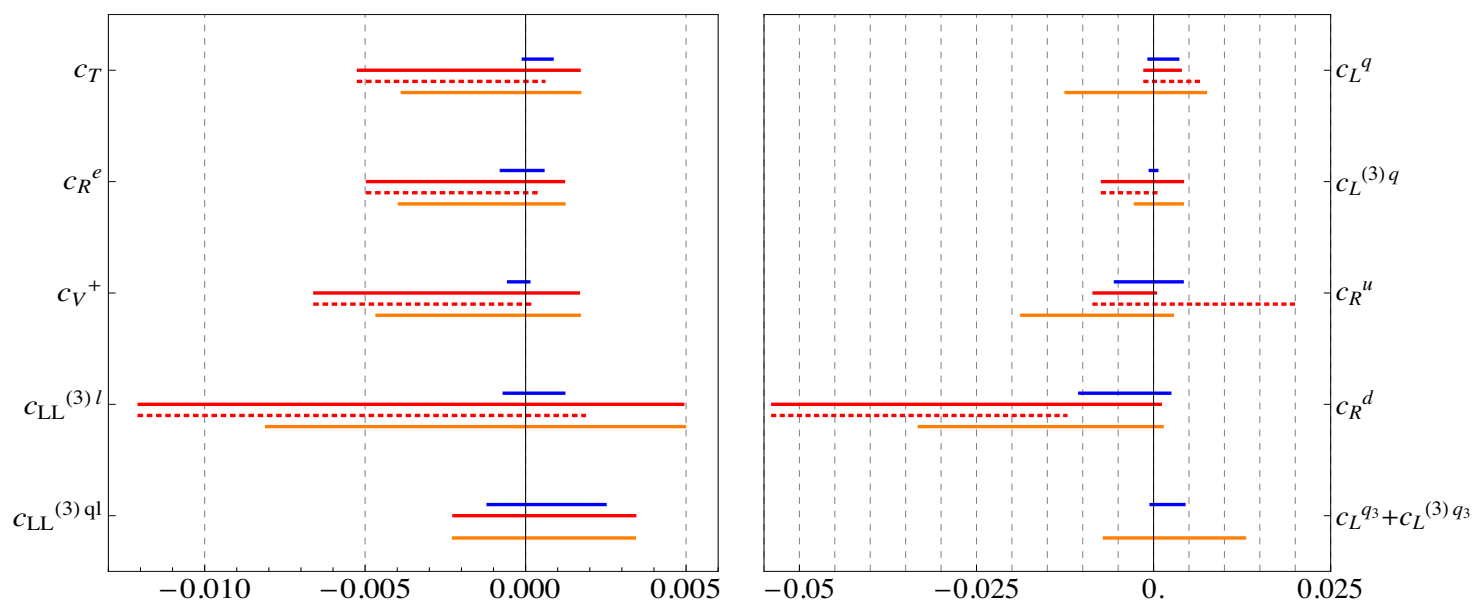

Figure 1. Bounds on the Wilson coefficients of eqs. (2.1), (2.5), (2.10) and (2.18), from LEP-I, SLC and KLOE data (and the necessary information from $p p \rightarrow l \bar{\nu}$ at the LHC - see appendix B). Solid red lines denote the $95 \%$ C.L. intervals after marginalizing over all other parameters (dashed lines do not include KLOE data); blue lines are obtained by setting all other coefficients to zero. The global fit including the operator of eq. (2.20) corresponds to the orange lines.

\section{Conclusions}

We have made a first step towards a complete SM fit, focusing here on EWSB effects in gauge-bosons and Higgs physics. We have characterized all possible deformations from SM physics using the Wilson coefficients of the independent dimension- 6 operators, the relevant ones for our analysis given in table 1. Assuming flavor-universality (but also including the top-quark operators separately) and taking as the input data $m_{Z}, G_{F}, \alpha_{\mathrm{em}}$ and $m_{f}, m_{h}$, we have grouped the operators according to their impact on the different experimental data.

In a first group we have the operators that can affect the gauge-boson propagators and their couplings to fermions. These receive strong constraints from well-measured quantities, mainly the $Z$-pole observables at LEP-I and SLC, the $W$ mass at Tevatron, together with the check of the CKM unitarity from low-energy data. The constraints on the corresponding Wilson coefficients from a global fit are summarized in figure 1 . To stress the correlation between the various operators, we compare the $95 \%$ C.L. contours obtained by marginalizing all other coefficients (in red) with the contours obtained by setting all other coefficients to zero (in blue). More data, such as the low-energy determination of the $\nu$-nucleon and $\nu$-e scattering or atomic parity violation experiments, could be added to our analysis. Nevertheless, due to their poorer resolution, we do not expect these data to affect substantially our results.

In a second group we have operators affecting triple gauge-boson vertices, that contrary to the previous group, receive milder constraints. Our basis is suitable for treating separately these two groups, while other bases, such as the one of ref. [11] used in the fit of ref. [7], makes this separation more difficult, due to strong correlations between bounds on different Wilson coefficients. We have 3 (combinations of) Wilson coefficients parametrizing these deviations, given in eq. (2.23). Using the 2-parameter fit of LEP-II [17] where 

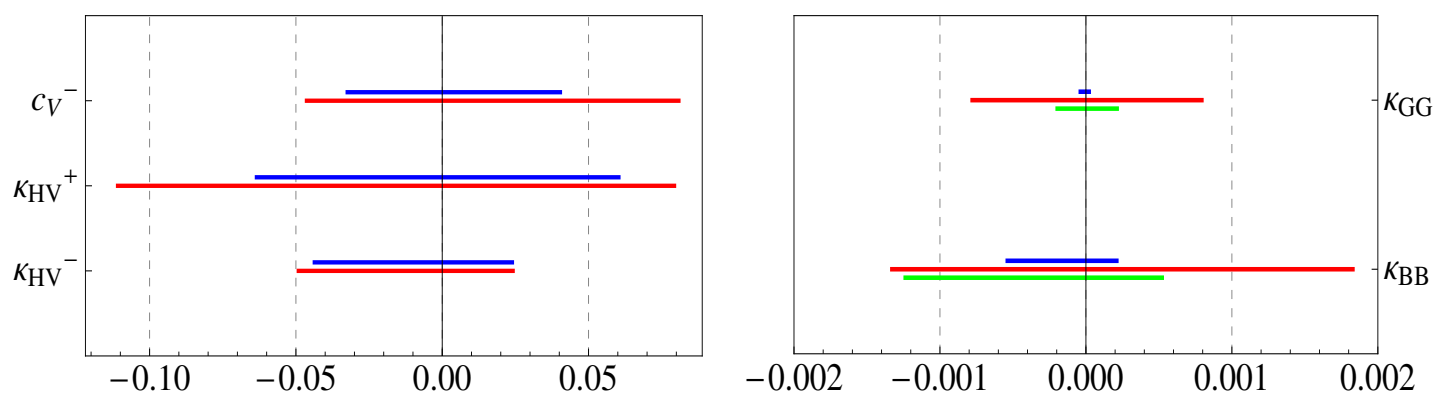

Figure 2. Left: bounds on some of the Wilson coefficients of eq. (2.23) from LEP-II TGC data and $B R(h \rightarrow Z \gamma)$. Right: bounds on some of the Wilson coefficients of eq. (3.1) from measurements of the Higgs BR at the LHC. Color code as in figure 1, with green lines including the theoretical prior on the coefficients $c_{H}$ and $c_{y_{f}}$ that cannot introduce modifications larger than $50 \%$ on SM predictions.

$\lambda_{\gamma}$ (and therefore $\kappa_{3 W}$ ) is neglected, we have presented bounds on two of them, eq. (2.28). Even if we allow for a nonzero $\lambda_{\gamma}$, LEP-II data is expected to be able to constrain all the 3 coefficients at the per-cent level [26], but a combined three-parameter fit is still not available. In figure 2 we show our results as bounds on the two coefficients $c_{V}^{-}$and $\kappa_{H V}^{+}$ (once the data on $B R(h \rightarrow Z \gamma)$ is also used to constrain the coefficient $\kappa_{H V}^{-}$).

Finally, there are the operators that only affect Higgs physics. These are $8 \mathrm{CP}$-even operators (for a single family) that can affect, independently, the Higgs $B R$ to fermions, $\gamma \gamma$ and $Z \gamma$, as well as production cross-sections both in the gluon-gluon and vector-boson fusion modes (and also the triple-Higgs coupling). In figure 2 we present the modelindependent constraints on the Wilson coefficients entering in the $h \gamma \gamma$ and $h G G$ effectivecouplings. These are very severe bounds as the BSM contributions enter at the tree-level and can then compete with the SM contributions arising at the one-loop level. Constraints on other Wilson coefficients, $c_{H}$ and $c_{y_{f}}$, are at present very mild and therefore not shown. The Wilson coefficient $\kappa_{H V}^{-}$enters in $B R(h \rightarrow Z \gamma)$ and is constrained, as we said, only form the present experimental bound $B R(h \rightarrow Z \gamma) \lesssim 10 \times B R(h \rightarrow Z \gamma)_{\mathrm{SM}}[32,33]$, leaving still room for large deviations from the SM.

Having determined all Wilson coefficients, any other (Higgs) process will depend on the above physics and therefore their BSM effects can be indirectly constrained. In particular, we have studied $h \rightarrow V \bar{f} f$. Neglecting corrections tightly bounded from the first group of observables, we have shown that the different form-factors of the $h \rightarrow V \bar{f} f$ amplitude are related with TGC and $B R(h \rightarrow Z \gamma)$. These latter are at present already constrained, and in the future LHC can considerably improve these bounds, being more competitive than looking for deviations in the $h \rightarrow V \bar{f} f$ decays. Similar arguments apply to CPodd operators.

Possible deviations in the approximate relations derived here, eq. (3.18), eq. (3.20) and eq. (4.9), would imply the breakdown of our assumptions, hinting possibly towards a nonlinear realization of EWSB, in which $h$ is not part of a Higgs doublet and no connection between Higgs and gauge-boson physics can be made. 


\section{Acknowledgments}

We are particularly grateful to M. Montull for collaboration in the early phase of this project. We also thank M. Carrer, J. Elias-Miro, J.R. Espinosa, E. Masso for discussions. FR thanks A. Avetisyan, O. Matsedonskii, A. Thamm and in particular K. Kotov for discussions about the analysis of appendix B. The work of AP was partly supported by the projects FPA2011-25948, 2009SGR894 and ICREA Academia Program, while FR acknowledges support from the Swiss National Science Foundation, under the Ambizione grant PZ00P2 136932 and thanks IFAE, Barcelona, for hospitality. We are grateful to the Galileo Galilei Institute for Theoretical Physics for the hospitality and the INFN for partial support during the completion of this work.

\section{A Corrections to $V \bar{f} f$ couplings and $Z$-pole observables}

The couplings between the $Z$-boson and fermions are altered as

$$
g_{Z}^{f}=g_{Z}^{f, \mathrm{SM}}+\delta g_{Z}^{f}+\delta g_{Z}^{f, \text { uni }},
$$

where

$$
g_{Z}^{f, \mathrm{SM}}=m_{Z}\left(\sqrt{2} G_{F}\right)^{1 / 2}\left(T_{L f}^{3}-Q_{f} s_{\theta_{W}}^{2}\right),
$$

is the SM value, $T_{L f}^{3}$ and $Q_{f}$ are respectively the weak-isospin and charge of the fermion $f=$ $\left\{f_{L}, f_{R}\right\}$, and $s_{\theta_{W}}^{2} \equiv\left(1-\sqrt{1-2^{3 / 2} \pi \alpha_{\mathrm{em}} / m_{Z}^{2} G_{F}}\right) / 2$. We have divided the corrections into a fermion-specific part $\delta g_{Z}^{f}$,

$$
\delta g_{Z}^{f_{L}}=m_{Z}\left(\sqrt{2} G_{F}\right)^{1 / 2}\left(T_{L, f}^{3} c_{L}^{(3) f}-\frac{c_{L}^{f}}{2}\right), \quad \delta g_{Z}^{f_{R}}=-m_{Z}\left(\sqrt{2} G_{F}\right)^{1 / 2} \frac{c_{R}^{f}}{2}
$$

(notice that for leptons $\delta g_{Z}^{l_{L}}=0$ in our basis) and a universal part,

$$
\delta g_{Z}^{f, \text { uni }}=-m_{Z}\left(\sqrt{2} G_{F}\right)^{1 / 2} Q_{f} \delta s_{\theta_{W}}^{2}+\frac{g_{Z}^{f}}{2}\left(\hat{T}-\frac{\delta G_{F}}{G_{F}}\right),
$$

which includes modifications to the wave-function of the $Z$-boson, $\delta \Pi_{Z}^{\prime}\left(m_{Z}^{2}\right)=2 \hat{S} s_{\theta_{W}}^{2}$, corrections to the input parameters, and contributions to $\Pi_{\gamma Z}\left(m_{Z}^{2}\right)$ through

$$
\delta s_{\theta_{W}}^{2}=\frac{1}{c_{2 \theta_{W}}}\left[s_{\theta_{W}}^{2} \hat{S}-\frac{s_{2 \theta_{W}}^{2}}{4}\left(\hat{T}-\frac{\delta G_{F}}{G_{F}}\right)\right] .
$$

This is enough to compute the corrections to the observables that we use in the fit (the uncorrelated subsets $\left\{A_{l}, R_{l}, \sigma_{\text {had }}^{0}, \Gamma_{Z}\right\}$ and $\left\{R_{b}, R_{c}, A_{F B}^{0, b}, A_{F B}^{0, c}, A_{b}, A_{c}\right\}$ from ref. [16]):

$$
A_{l} \equiv \frac{\left(g_{Z}^{l_{L}}\right)^{2}-\left(g_{Z}^{l_{R}}\right)^{2}}{\left(g_{Z}^{l_{L}}\right)^{2}+\left(g_{Z}^{l_{R}}\right)^{2}}, \quad R_{l} \equiv \frac{\Gamma_{Z}^{\mathrm{had}}}{\Gamma_{Z}^{l}}, \quad \sigma_{\mathrm{had}}^{0} \equiv \frac{12 \pi}{m_{Z}^{2}} \frac{\Gamma_{Z}^{l} \Gamma_{Z}^{\mathrm{had}}}{\Gamma_{Z}^{2}}
$$


and

$$
R_{q}=\frac{\left(g_{Z}^{q_{L}}\right)^{2}+\left(g_{Z}^{q_{R}}\right)^{2}}{3\left[\left(g_{Z}^{d_{L}}\right)^{2}+\left(g_{Z}^{d_{R}}\right)^{2}\right]+2\left[\left(g_{Z}^{u_{L}}\right)^{2}+\left(g_{Z}^{u_{R}}\right)^{2}\right]}, \quad A_{q}=\frac{\left(g_{Z}^{q_{L}}\right)^{2}-\left(g_{Z}^{q_{R}}\right)^{2}}{\left(g_{Z}^{q_{L}}\right)^{2}+\left(g_{Z}^{q_{R}}\right)^{2}}, \quad A_{F B}^{0, q}=\frac{3}{4} A_{q} A_{l},
$$

where $q=b, c$ at LEP. The partial widths are defined as,

$$
\begin{array}{rlrl}
\Gamma_{Z}^{l} & =\frac{m_{Z}}{24 \pi}\left[\left(g_{Z}^{l_{L}}\right)^{2}+\left(g_{Z}^{l_{R}}\right)^{2}\right], \Gamma_{Z}^{\mathrm{had}} & =\frac{m_{Z}}{24 \pi}\left(3 N_{c}\left[\left(g_{Z}^{d_{L}}\right)^{2}+\left(g_{Z}^{d_{R}}\right)^{2}\right]+2 N_{c}\left[\left(g_{Z}^{u_{L}}\right)^{2}+\left(g_{Z}^{u_{R}}\right)^{2}\right]\right), \\
\Gamma_{Z}^{\nu} & =\frac{m_{Z}}{24 \pi}\left(g_{Z}^{\nu}\right)^{2}, & \Gamma_{Z} & =\Gamma_{Z}^{\mathrm{had}}+3 \Gamma_{Z}^{l}+3 \Gamma_{Z}^{\nu} .
\end{array}
$$

Notice that the universal corrections due to $\delta \Pi_{Z}^{\prime}\left(m_{Z}^{2}\right)$ cancel out from the observables eq. (A.6), but enter in the total width (and similarly for the partial widths) which is corrected as

$$
\frac{\delta \Gamma_{Z}}{\Gamma_{Z}}=2 \frac{\sum_{f} g_{Z}^{f}\left(\delta g_{Z}^{f, \text { uni }}+\delta g_{Z}^{f}\right)}{\sum_{f}\left(g_{Z}^{f}\right)^{2}} .
$$

Finally, the couplings of $W$-bosons to fermions are modified as

$$
g_{W}^{f}=g_{W}^{f, \mathrm{SM}}+\delta g_{W}^{f}+\delta g_{W}^{f, \text { uni }},
$$

where

$$
g_{W}^{f, \mathrm{SM}}=\frac{m_{W} G_{F}^{1 / 2}}{2^{1 / 4}}, \quad \frac{\delta g_{W}^{f}}{g_{W}^{f}}=c_{L}^{(3) f}, \quad \frac{\delta g_{W}^{f, \text { uni }}}{g_{W}^{f}}=\frac{\delta m_{W}}{m_{W}}-\frac{\delta G_{F}}{2 G_{F}} .
$$

\section{B $q \bar{q}^{\prime} l \bar{\nu}$ contact-interactions at the LHC}

Since the fermion-fermion scattering amplitude mediated by contact-interactions grows with energy, these can be tested with accuracy at the LHC. We are interested here to put a bound on the 4 -fermion operator $\mathcal{O}_{L L}^{(3)} q l$. This operator affects in particular the cross-section of $p p \rightarrow l \bar{\nu}$ that has been measured at the LHC. Nevertheless, the only LHC analysis [22] has been on the helicity non-conserving (HNC) operator $\left(Q_{L}^{r} \bar{u}_{R}\right) \epsilon_{r s}\left(L_{L}^{s} \bar{e}_{R}\right)$, which also modifies $p p \rightarrow l \bar{\nu}$. Contrary to $\mathcal{O}_{L L}^{(3) q l}$, however, the HNC operator does not interfere with the SM contribution, so that the results of ref. [22] cannot be used for $\mathcal{O}_{L L}^{(3)} q l$ and a dedicated analysis is necessary.

A signal sample needs to be considered for every value of $c_{L L}^{(3)} q l$ and then compared with the data. Other operators entering in $p p \rightarrow l \bar{\nu}$, such as the HNC operator, are suppressed by small Yukawa couplings under the MFV assumption, that we consider here, and can be neglected. We simulate the effects of $\mathcal{O}_{L L}^{(3)} q l$ by integrating out a heavy $W^{\prime}$-boson, implemented with FeynRules [43] and generate parton-level events with MadGraph [44]. We divide the signal and the data in 3 regions, according to the transverse mass of electron and neutrino: $M_{T} \in[1,1.5] \mathrm{TeV}, M_{T} \in[1.5,2] \mathrm{TeV}$ and $M_{T}>2 \mathrm{TeV}$, which we treat as statistically independent. Ref. [22] reports, respectively, the observation of 22, 0 and 1 events in these regions. We compute C.L. contours, assuming a Poissonian distribution around the signal+background and treating errors as nuisances (an estimated 5\% systematic error 


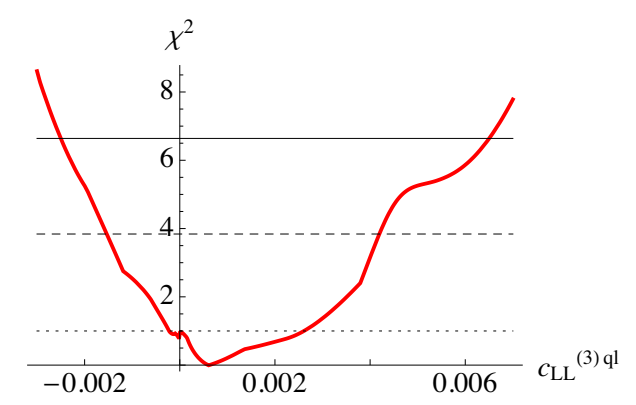

Figure 3. The $\chi^{2}$-distribution for the Wilson coefficient of the operator $\mathcal{O}_{L L}^{(3) q l}$.

in the signal plus a $4.4 \%$ in the luminosity are summed in quadrature). We checked that such estimates reproduced satisfactorily the results in the case of the HNC model. Our results, in form of the $\chi^{2}$-distribution, are summarized in figure 3 and imply

$$
-0.001 \lesssim c_{L L}^{(3)} q l \lesssim 0.004 \quad \text { at } 95 \% \text { C.L. . }
$$

Combining it with the muon channel can give better limits in flavour-universal BSM models, as the ones considered here. Notice that bounds on $c_{L L}^{(3)} q l$ from the differential distribution of $p p \rightarrow l^{+} l^{-}$at the LHC [24] are weakened once the contributions from other operators are taken into account. Therefore, eq. (B.1) provides at present the best model-independent bound on this Wilson coefficient.

Open Access. This article is distributed under the terms of the Creative Commons Attribution License (CC-BY 4.0), which permits any use, distribution and reproduction in any medium, provided the original author(s) and source are credited.

\section{References}

[1] J. Elias-Miro, J. Espinosa, E. Masso and A. Pomarol, Higgs windows to new physics through $D=6$ operators: constraints and one-loop anomalous dimensions, JHEP 11 (2013) 066 [arXiv: 1308.1879] [INSPIRE].

[2] Z. Han and W. Skiba, Effective theory analysis of precision electroweak data, Phys. Rev. D 71 (2005) 075009 [hep-ph/0412166] [INSPIRE].

[3] F. del Aguila and J. de Blas, Electroweak constraints on new physics, Fortsch. Phys. 59 (2011) 1036 [arXiv:1105.6103] [INSPIRE].

[4] E. Masso and V. Sanz, Limits on Anomalous Couplings of the Higgs to Electroweak Gauge Bosons from LEP and LHC, Phys. Rev. D 87 (2013) 033001 [arXiv:1211.1320] [InSPIRE].

[5] R. Contino, M. Ghezzi, C. Grojean, M. Muhlleitner and M. Spira, Effective Lagrangian for a light Higgs-like scalar, JHEP 07 (2013) 035 [arXiv:1303.3876] [INSPIRE].

[6] B. Dumont, S. Fichet and G. von Gersdorff, A Bayesian view of the Higgs sector with higher dimensional operators, JHEP 07 (2013) 065 [arXiv: 1304.3369] [INSPIRE].

[7] B. Grinstein, C.W. Murphy and D. Pirtskhalava, Searching for new physics in the three-body decays of the Higgs-like particle, JHEP 10 (2013) 077 [arXiv:1305.6938] [INSPIRE]. 
[8] M. Ciuchini, E. Franco, S. Mishima and L. Silvestrini, Electroweak Precision Observables, New Physics and the Nature of a 126 GeV Higgs Boson, JHEP 08 (2013) 106 [arXiv: 1306.4644] [INSPIRE].

[9] G. Giudice, C. Grojean, A. Pomarol and R. Rattazzi, The strongly-interacting light Higgs, JHEP 06 (2007) 045 [hep-ph/0703164] [INSPIRE].

[10] J. Elias-Miró, J. Espinosa, E. Masso and A. Pomarol, Renormalization of dimension-six operators relevant for the Higgs decays $h \rightarrow \gamma \gamma, \gamma Z$, JHEP 08 (2013) 033 [arXiv: 1302.5661] [INSPIRE].

[11] B. Grzadkowski, M. Iskrzynski, M. Misiak and J. Rosiek, Dimension-Six Terms in the Standard Model Lagrangian, JHEP 10 (2010) 085 [arXiv: 1008.4884] [INSPIRE].

[12] W. Buchmüller and D. Wyler, Effective Lagrangian Analysis of New Interactions and Flavor Conservation, Nucl. Phys. B 268 (1986) 621 [INSPIRE].

[13] G. D'Ambrosio, G. Giudice, G. Isidori and A. Strumia, Minimal flavor violation: an effective field theory approach, Nucl. Phys. B 645 (2002) 155 [hep-ph/0207036] [INSPIRE].

[14] M.E. Peskin and T. Takeuchi, A new constraint on a strongly interacting Higgs sector, Phys. Rev. Lett. 65 (1990) 964 [INSPIRE].

[15] R. Barbieri, A. Pomarol, R. Rattazzi and A. Strumia, Electroweak symmetry breaking after LEP-1 and LEP-2, Nucl. Phys. B 703 (2004) 127 [hep-ph/0405040] [INSPIRE].

[16] ALEPH, DELPHI, L3, OPAL, SLD, LEP Electroweak Working Group, SLD Electroweak Group and SLD Heavy Flavour Group collaborations, S. Schael et al., Precision electroweak measurements on the $Z$ resonance, Phys. Rept. 427 (2006) 257 [hep-ex/0509008] [INSPIRE].

[17] LEP, ALEPH, DELPHI, L3, OPAL and LEP TGC Working Group collaborations, $A$ combination of preliminary results on gauge boson couplings measured by the LEP experiments, LEPEWWG/TGC/2003-01 (2004).

[18] M. Baak et al., The Electroweak Fit of the Standard Model after the Discovery of a New Boson at the LHC, Eur. Phys. J. C 72 (2012) 2205 [arXiv:1209.2716] [InSPIRE].

[19] A. Arbuzov et al., ZFITTER: a semi-analytical program for fermion pair production in $e^{+} e^{-}$ annihilation, from version 6.21 to version 6.42, Comput. Phys. Commun. 174 (2006) 728 [hep-ph/0507146] [INSPIRE].

[20] ALEPH, DELPHI, L3, OPAL and LEP Electroweak collaborations, S. Schael et al., Electroweak Measurements in Electron-Positron Collisions at W-Boson-Pair Energies at LEP, Phys. Rept. 532 (2013) 119 [arXiv: 1302.3415] [INSPIRE].

[21] M. Antonelli et al., An evaluation of $\left|V_{u s}\right|$ and precise tests of the Standard Model from world data on leptonic and semileptonic kaon decays, Eur. Phys. J. C 69 (2010) 399 [arXiv: 1005.2323] [INSPIRE].

[22] CMS collaboration, Search for new physics in final states with a lepton and missing transverse energy in pp collisions at the LHC, Phys. Rev. D 87 (2013) 072005 [arXiv: 1302.2812] [INSPIRE].

[23] O. Domenech, A. Pomarol and J. Serra, Probing the SM with Dijets at the LHC, Phys. Rev. D 85 (2012) 074030 [arXiv: 1201.6510] [INSPIRE].

[24] J. de Blas, M. Chala and J. Santiago, Global Constraints on Lepton-Quark Contact Interactions, Phys. Rev. D 88 (2013) 095011 [arXiv:1307.5068] [INSPIRE].

[25] K. Hagiwara, R. Peccei, D. Zeppenfeld and K. Hikasa, Probing the Weak Boson Sector in $e^{+} e^{-} W^{+} W^{-}$, Nucl. Phys. B 282 (1987) 253 [INSPIRE]. 
[26] DELPHI collaboration, J. Abdallah et al., Measurements of CP-conserving Trilinear Gauge Boson Couplings $W W V(V=\gamma, Z)$ in $e^{+} e^{-}$Collisions at LEP2, Eur. Phys. J. C 66 (2010) 35 [arXiv: 1002.0752] [INSPIRE].

[27] ATLAS collaboration, Combined coupling measurements of the Higgs-like boson with the ATLAS detector using up to $25 \mathrm{fb}^{-1}$ of proton-proton collision data, ATLAS-CONF-2013-034 (2013).

[28] CMS collaboration, Updated measurements of the Higgs boson at 125 GeV in the two photon decay channel, CMS-PAS-HIG-13-001 (2013).

[29] CMS collaboration, Properties of the Higgs-like boson in the decay H to ZZ to $4 \mathrm{l}$ in $p p$ collisions at $\sqrt{s}=7$ and 8 TeV, CMS-PAS-HIG-13-002 (2013).

[30] CMS collaboration, Evidence for a particle decaying to $W+W$ - in the fully leptonic final state in a standard model Higgs boson search in pp collisions at the LHC, CMS-PAS-HIG-13-003 (2013).

[31] A. Falkowski, F. Riva and A. Urbano, Higgs at last, JHEP 11 (2013) 111 [arXiv: 1303.1812] [INSPIRE].

[32] CMS collaboration, Search for a Higgs boson decaying into a $Z$ and a photon in pp collisions at $\sqrt{s}=7$ and 8 TeV, Phys. Lett. B 726 (2013) 587 [arXiv:1307.5515] [INSPIRE].

[33] ATLAS collaboration, Search for the Standard Model Higgs boson in the $H \rightarrow Z \gamma$ decay mode with pp collisions at $\sqrt{s}=7$ and 8 TeV, ATLAS-CONF-2013-009 (2013).

[34] S. Choi, D. Miller, M. Muhlleitner and P. Zerwas, Identifying the Higgs spin and parity in decays to Z pairs, Phys. Lett. B 553 (2003) 61 [hep-ph/0210077] [InSPIRE].

[35] Q.-H. Cao, C. Jackson, W.-Y. Keung, I. Low and J. Shu, The Higgs Mechanism and Loop-induced Decays of a Scalar into Two Z Bosons, Phys. Rev. D 81 (2010) 015010 [arXiv:0911.3398] [INSPIRE].

[36] Y. Gao et al., Spin determination of single-produced resonances at hadron colliders, Phys. Rev. D 81 (2010) 075022 [arXiv: 1001.3396] [INSPIRE].

[37] D. Stolarski and R. Vega-Morales, Directly Measuring the Tensor Structure of the Scalar Coupling to Gauge Bosons, Phys. Rev. D 86 (2012) 117504 [arXiv: 1208.4840] [INSPIRE].

[38] G. Isidori, A.V. Manohar and M. Trott, Probing the nature of the Higgs-like Boson via $h \rightarrow V \mathcal{F}$ decays, Phys. Lett. B 728 (2014) 131 [arXiv:1305.0663] [InSPIRE].

[39] G. Isidori and M. Trott, Higgs form factors in Associated Production, arXiv:1307.4051 [INSPIRE].

[40] LHC Higgs Cross Section Working Group collaboration, A. David et al., LHC HXSWG interim recommendations to explore the coupling structure of a Higgs-like particle, arXiv: 1209.0040 [INSPIRE].

[41] S. Dawson, S.K. Gupta and G. Valencia, CP violating anomalous couplings in $W \gamma$ and $Z \gamma$ production at the LHC, Phys. Rev. D 88 (2013) 035008 [arXiv: 1304.3514] [InSPIRE].

[42] M. Carrel, Comment on non-compact composite Higgs models and constraints on the phenomenology of dimension 6 operators, MSc Thesis, EPFL, Lausanne Switzerland (2013).

[43] N.D. Christensen and C. Duhr, FeynRules - Feynman rules made easy, Comput. Phys. Commun. 180 (2009) 1614 [arXiv:0806.4194] [INSPIRE].

[44] J. Alwall, M. Herquet, F. Maltoni, O. Mattelaer and T. Stelzer, MadGraph 5: going beyond, JHEP 06 (2011) 128 [arXiv:1106.0522]. 